Anatoliy G. Lozhkovskiy', Ehtiram I. Kerimov', Oleq V. Verbanov $\quad$ DOI: 10.25045/jpit.v07.i1.03

${ }^{1,3}$ Odessa National Academy of Telecommunications O.S. Popov, Odessa, Ukraine

${ }^{2}$ Ministry of Telecommunications and high technologies, Baku, Azerbaijan

1,3aloshk@onat.edu.ua, ${ }^{2}$ kerimov@mincom.gov.az

\title{
CALCULATION CHARACTERISTICS OF THE SELF-SIMILAR TRAFFIC APPROXIMATED BY THE WEIBULL DISTRIBUTION
}

The article studies the methods to improve the accuracy of calculation of the characteristics of service quality in the networks with self-similar traffic through more precise location Hurst coefficient depending on the parameters of Weibull distribution form. Since the self-similar traffic (time interval between the requests) best describes the Weibull distribution, it is provided a new formula for the calculation of the self-similarity coefficient of traffic. The calculation of the characteristics of service quality can be performed based on the Norros formula, which is valid for the model $f B M / D / 1 / \infty$.

Keywords: telecommunication systems and networks, calculation and projecting methods, selfsimilar traffic, Weibull distribution.

\section{Introduction}

In the face of the popularity of the self-similar traffic, a number of challenges in assessing the quality of service in packet-based network remain unsolved. Due to the lack of a rigorous theoretical framework that can complement the classical queueing theory in the projecting of the packet-based network with self-similar traffic, there is no reliable and accepted method for calculating the parameters and quality of information distribution systems in terms of self-similarity effect. In [1-4] it is shown that the service quality degrades when self-similarity exists in the incoming requirements' flow with increasing intensity load $\rho$, but not as much as expected in the case of the Norros method [5]. The discrepancy between the results of modelling and evaluations obtained by the Norros method is hundreds of percent [1]. Obviously, the evaluation obtained by the Norros is significantly overstated, and that requires providing more accurate solution.

\section{Problem statement}

This article aims at improving the accuracy of the calculation of the characteristics of service quality by obtaining a new formula for calculating the coefficient of self-similarity of the traffic, depending on the parameter of the form of the Weibull distribution, as the self-similar traffic (time interval between the applications or stream packets) can be well described by the Weibull distribution.

For single-server system with an infinite queuing and constant service time (model $f B M / D / 1 / \infty)$, this tough solution is known as the Norros formula $[5,6]$ :

$$
N=\frac{(1-\rho)^{\frac{H}{H-1}}}{\rho^{\frac{0.5}{H-1}}}
$$

$\mathrm{N}$ - denotes the average number of requests in the system, which must not be exceeded, i.e. it is the higher number of customers in the system $f B M / D / 1 / \infty$, and $\mathrm{H}$ - denotes the coefficient of selfsimilarity of the packet traffic, called the Hurst coefficient.

The Hurst method reveals the properties in the statistical data of packet traffic such as clustering, a tendency to follow the trend direction (persistence) and fast intermittency of successive values of traffic intensity (intensity bursts, resulting in a burst), strong aftereffect and memory, fractal (selfsimilarity), the presence of periodic and aperiodic cycles (due to the nature of the used protocols of packet traffic transmission). 
The modelling results shown in Figure 1 indicate that the service quality degrades, when selfsimilarity exists in the incoming requirements' flow with increasing intensity load $\rho$, but not as much as expected in the case of the Norros method. The discrepancy between the modelling results (indicated by "+") and the estimations derived from the formula (1) (shown in dashed lines), can reach hundreds of percent. The evaluation obtained by the Norros significantly is overstated, and requires more accurate solution.

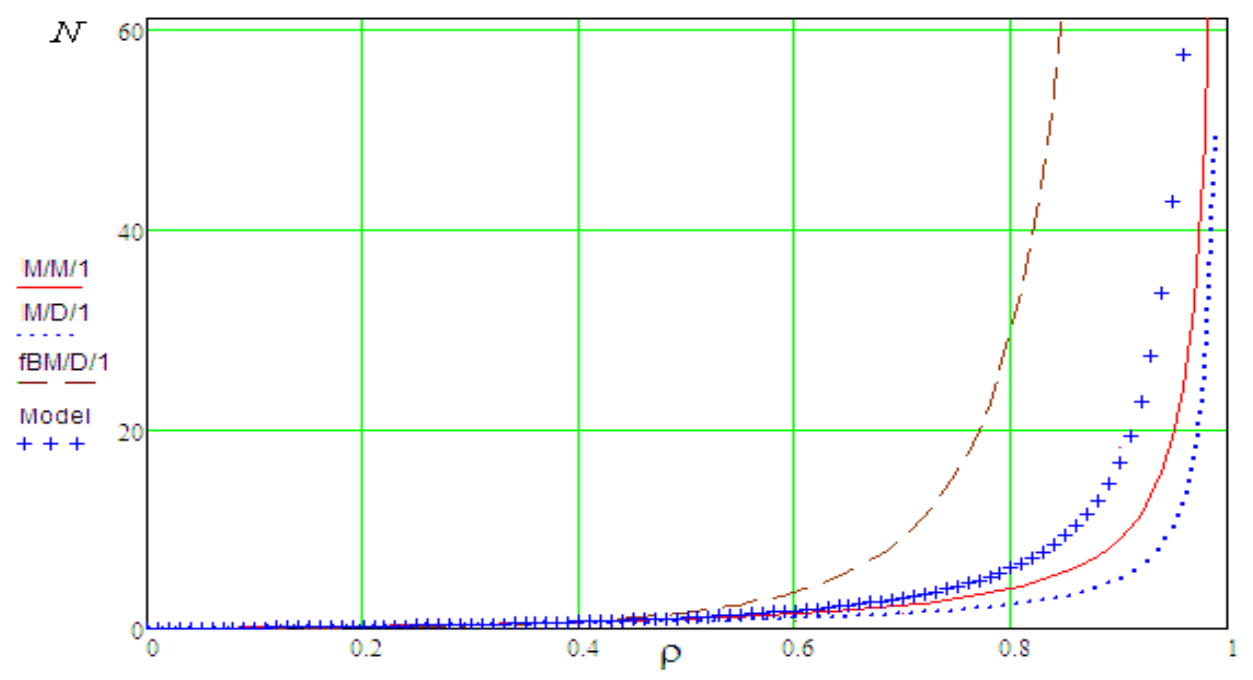

Fig. 1. $N$ modelling in the model $f B M / D / 1 / \infty$ when $H=0.7$

Burst nature of generated traffic contributes to its adequacy, to the real nature of the traffic, in the multiservice networks. Where a wide range of load transmission rates is heterogeneous since the transmission of the flows of different applications and services provide the same network with a single protocol and control laws. The sources of the particular service are characterized by the maximum and average transmission rates, i.e., the burstness and the mean duration of the peak load. For example, burstness for voice services may possibly be due to pauses in the conversation.

\section{Problem solution}

The method of Mandelbrot for forming a self-similar flow is known [7]. It is based on the superposition of several independent ON/OFF sources, which have the same distribution, and the intervals between the ON and OFF periods of which have the Noah effect. Noah effect, in the distribution of durations of ON/OFF time series, is the base for modeling of self-similar traffic. Noah Effect is synonymous with infinite dispersion syndrome. To achieve the Noah effect, we use Pareto or Weibull distribution, often called "long-tailed distribution". The presence of the "long tail" in the distribution provides traffic burst, as the probability of long intervals between the events increases in the distribution, and the probability of very short intervals increases for their compensation, as well.

The density of the Weibull distribution is set by the function:

$$
\lambda_{0} a x^{a-1} e^{-\lambda_{0} x^{a}} \text {. }
$$

where $\mathrm{a}$-denotes the shape parameter; $\lambda 0$ - scale parameter.

In the practical modelling of self-similar traffic, for example, using the algorithm [8], the Pareto distribution is obtained by moving from a uniform distribution by the inverse functions:

$$
X_{i}=\left(-\frac{1}{\lambda 0} \ln U_{i}\right)^{1 / a}
$$


Where $X_{i}$-denotes the $i$-th interval between the requests of flow; $U$ - a random number is uniformly distributed on the interval $[0,1]$. To ensure self-similar properties of the modelled traffic, it is necessary to set the values of the shape parameter, $a$ from 1 to 0 , which should ensure the values of the self-similarity Hurst coefficient in the range $\mathrm{H}=0.5 \ldots 1$, respectively.

It is believed [6] that the shape parameter $a$ of the Weibull distribution and Hurst coefficient $H$ are in the following relationship:

$$
H=\frac{2-a}{2} .
$$

However, the modelling results shown in Fig. 2 indicate that there is no any linear dependence (3) of Hurst coefficient $H$ on the shape parameter $a$ of the distribution for the Weibull distribution (and Pareto).

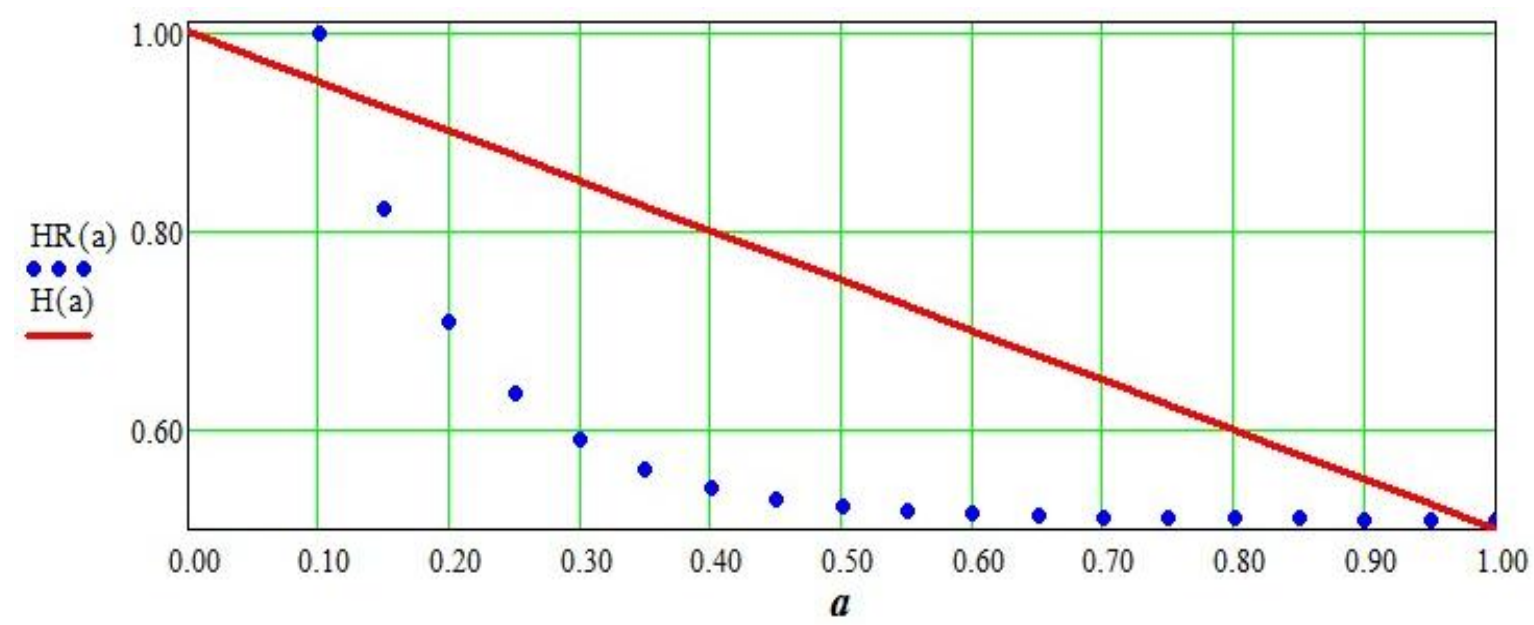

Fig. 2. Modelling self-similarity coefficient of $H R$ in model $f B M / D / 1 / \infty$

Fig. 2 shows that the actual coefficient of Hearst $H R$ (dotted curve) non-linearly depends on the shape parameter $a$ on the Weibull distribution (solid line), that is close to exponential. While the actual traffic statistics (time interval between requests or packets) is approximated by the Weibull distribution, and for the Norros formula (1), the self-similarity Hurst coefficient should be calculated not by the formula (3), but by the formula approximating the curve $H R$, shown in Fig. 2 with a dotted line. The accuracy of calculation (1) is significantly increased, since, for example, (3) shows that when $a=0.3$ the coefficient $H=0.85$, and actually $H=0.6$, which is less by $40 \%$.

According to the results of simulated modelling carried out with the use of the algorithm [8], for the calculation of Hurst traffic coefficient described by the Weibull distribution, the following formula is offered:

$$
H w=1,2 e^{-9 a}+0,51
$$

$a$ - denotes the shape parameter of the Weibull distribution. 


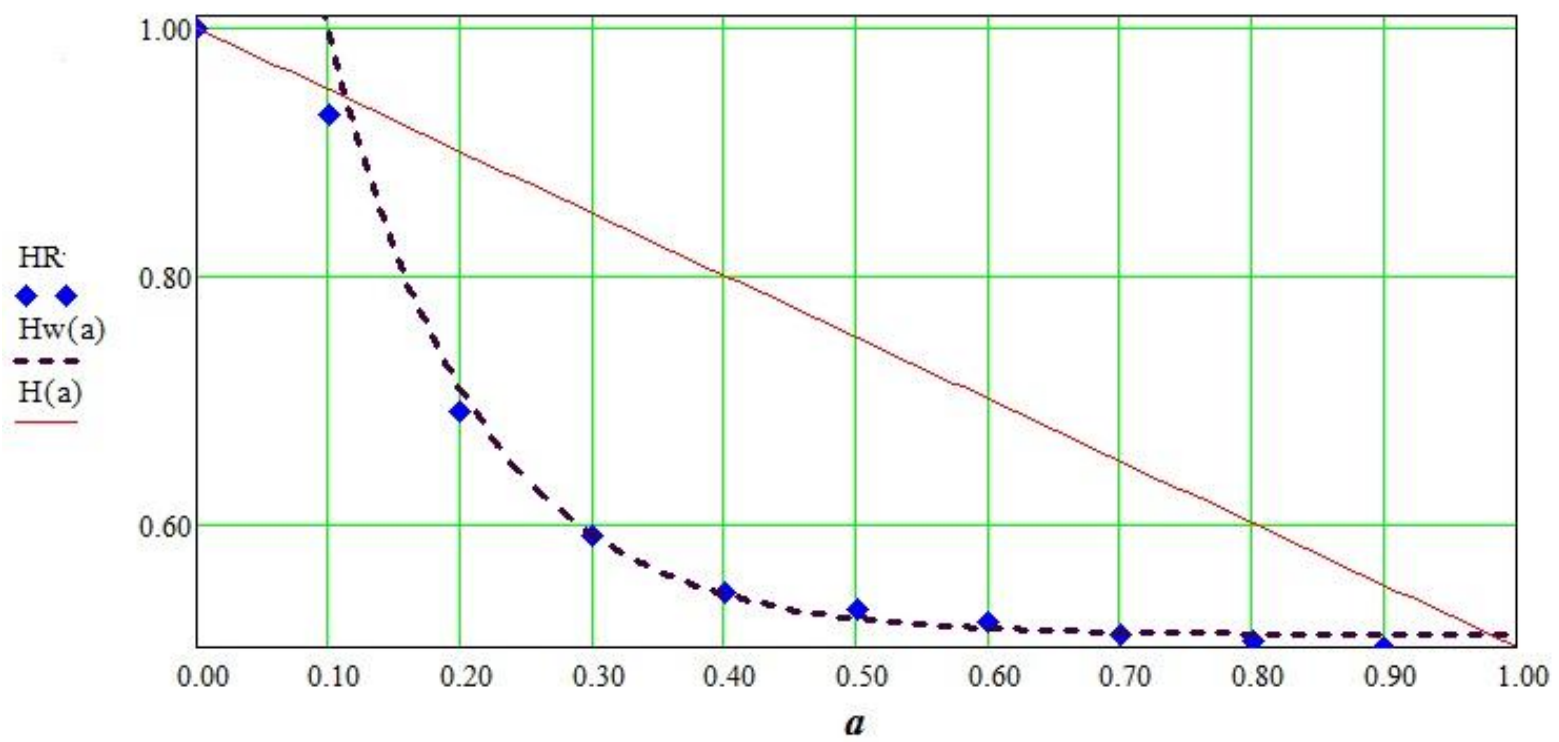

Fig. 3. Approximation of Hurst coefficient of $H R$ in the model $f B M / D / 1 / \infty$

Approximation (4) of the Hurst coefficient HR (dashed line), shown in Figure 3, although it is not fully comply with the curve of the real change of the Hurst coefficient, depending on the parameter of the form $a$ of the Weibull distribution, but it ensures the accuracy of the calculation of the characteristics of the service quality much higher than the calculations using the formula (3). The error of the calculation does not averagely exceed $10 \ldots 20 \%$.

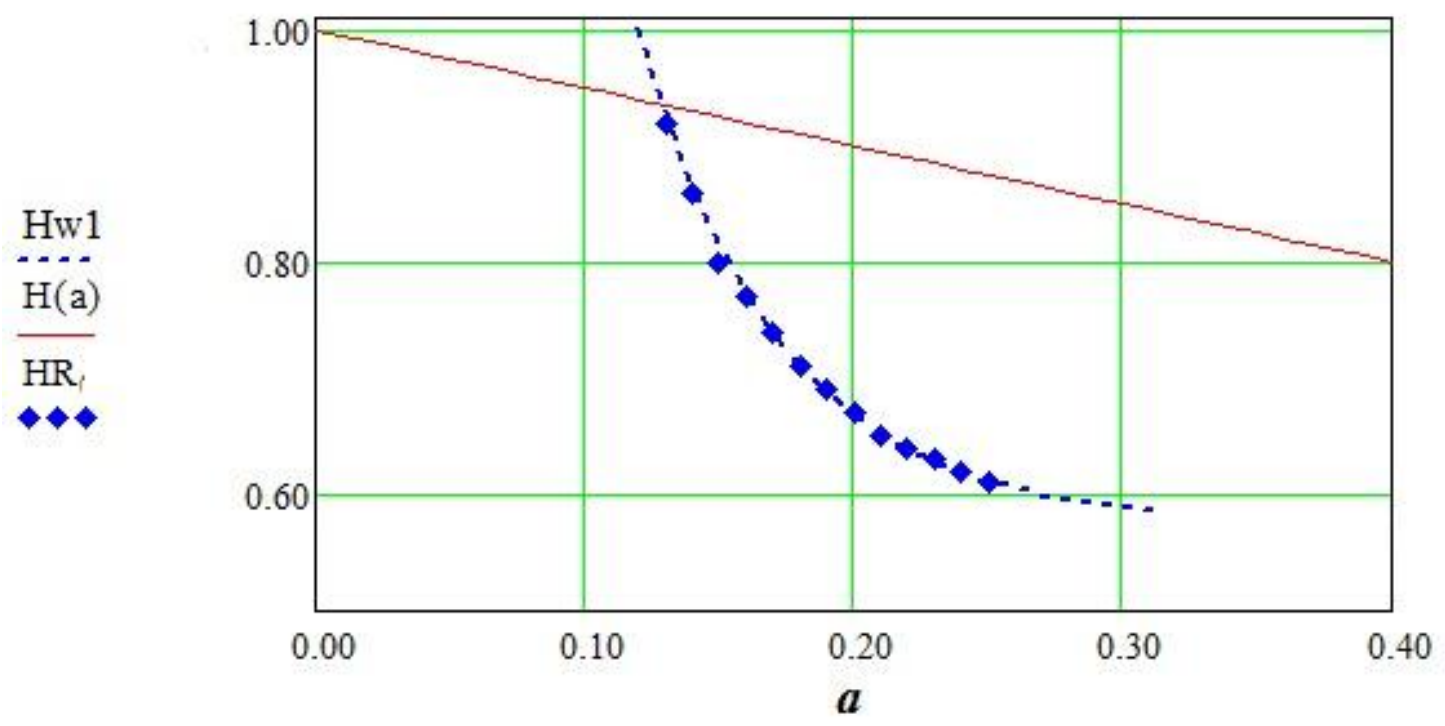

Fig. 4. Approximation of the Hurst coefficient $H R$ when $H=0,6 \ldots 0,9$

The Fig. 4 shows the approximation for the range of values of the coefficient $H=0,6 \ldots 0,9$ as

$$
H w 1=4,1 e^{-19 a}+0,57 \text {, }
$$

which provides more accurate calculation of the Hurst coefficient $H$, depending on the parameter of the form $a$ of the Weibull distribution. In this range there are the values of the coefficients $H$ of real self-similar traffic packet networks.

Taking into account this approximation while evaluating the characteristics of the quality of service it is sufficient to calculate only one of the characteristics through the parameter of the form 
$a$ of the Weibull distribution, for example, the average number of requests in the system $N$ by the formula (1). All remaining QoS characteristics are calculated by the following formulas, as the characteristics such as the average number of the requests in the queue $Q$, the average presence time of applications in the system $T$ and the average delay time of the requests in the system $W$, are associated with the known functional relations $N$ :

$$
Q=N-\rho, \quad T=\frac{N}{\rho}, \quad W=T-1 .
$$

\section{Conclusion}

It should be noted that this method allows us to calculate the characteristics of the quality of service of self-similar traffic described by the Weibull distribution, and in a single-channel system $f B M / D / 1 / \infty$ with discrete-time of service delivery it is much easier. This simplicity is due to the fact that, it is necessary to know only the parameter of the form $a$ of the Weibull distribution, and there is no need to calculate the self-similarity coefficient Hurst by the quite difficult and timeconsuming methods (for example, by R/S-statistics) for the traffic. All the given graphs show a significant difference between the real (5) and currently used linear dependence (3) of the selfsimilarity coefficient $H$ on the parameter of the form $a$ of the Weibull distribution in the system $f B M / D / 1 / \infty$ with the traffic described by this distribution. Using the real functional dependence of $H$ and $a$ can improve the accuracy of calculation of the characteristics of the quality of service.

\section{References}

1. Lozhkovskiy A.G. Comparative analysis of the methods for calculating the characteristics of quality of service in the self-similar flows in the network / A.G.Lozhkovskiy // Modelling and information technologies: ST. Sciences. pr. IPME IM. G.E. Pukhov National Academy of Sciences of Ukraine, K., 2008, No 47, pp.187-193.

2. Lozhkovskiy A.G., Verbanov O.V., Kaptur V.A., Kolchar V.M. Mathematical model of packet traffic // Bulletin of the National Technical University “KPI", 2011, No 9, pp.113-119.

3. Lozhkovskiy A.G., Hanifayev R.A. Estimation of the parameters of quality of service of self-similar traffic through entropy method // Naukovi pratsi ONAZ O.S.Popov, 2008, No 1, pp. 57-62.

4. Lozhkovskiy A.G., Verbanov O.V. Modeling of the multiservice packet networks traffic with the estimation of its self-similarity coefficient // Collection Naukova Pratzen ONAZI O.S. Popov, 2014, No 1, pp.70-76.

5. Norros Ilkka. A storage model with self-similar input, Queuing Systems, 1994, vol.16, no.3, pp.387-396.

6. Krylov V.V., Samokhvalova S.S. Teletraffic Theory and Its Applications. SPb.: BHVPetersburg, 2005, p.288.

7. Mandelbrot B. Fractal Geometry of Nature // Computing in mathematics, physics, biology; translated from English by Mandelbrot B., M.: Publishing House of the Institute of Computer Studies, 2002, p.655.

8. Lozhkovskiy A.G., Salmanov N.S., Verbanov O.V. Modelling of a multi-service system with queuing // Eastern European Journal of advanced technologies, 2007, No 3/6 (27), pp.72-76. 